\title{
Abgabe von medizinischen Kompressionsstrümpfen in der gefäßmedizinischen Praxis
}

\section{Rechtliche Beurteilung durch \\ Rechtsanwältin Patrizia \\ Gratwohl, M.A. HSG in Law \\ Zusammenfassung (Jürg Hafner, Präsident SGP, und Daniel Staub, Präsident USGG)}

Die Anmessung und Abgabe medizinischer Kompressionsstrümpfe in der gefäßmedizinischen Praxis mit Rückvergütung durch die obligatorischen Krankenpflegeversicherung (OKP) ist in der Schweiz seit Jahrzehnten unbeanstandeter Usus.

Seit der Inkraftsetzung der Revision der Mittel- und Gegenständeliste (MiGeL) vom 01.04.2019 wurde der SGP-Vorstand wiederholt informiert, dass Leistungsträger (Krankenkassen und weitere Krankenversicherer) medizinische Kompressionsstrümpfe, welche in der gefäßmedizinischen Praxis angemessen und abgegeben wurden, nicht mehr rückvergüteten.

Aus diesem Anlass beauftragten die SGP und die USGG Rechtsanwältin Patrizia Gratwohl mit einer rechtlichen Beurteilung (Memorandum). Frau RA lic. iur. P. Gratwohl ist Partnerin der Kanzlei Steinbrüchel Hüssy Rechtsanwälte, 8001 Zürich.

Die folgenden Zeilen fassen den Inhalt des Memorandums vom 01.03.2021 zusammen, welches wir auch auf unseren Websites aufschalten werden.

\section{KERNAUSSAGE}

Für die Abgabe respektive Verrechnung von Kompressionsstrümpfen zulasten der OKP durch die verschreibenden Ärzte ist kein Vertrag mit den Versicherern erforderlich. Dies ergibt sich in erster Linie aus der Gesetzessystematik, welche die Zulassung von Produkten aus der MiGeL für (A) die Ärzteschaft und (B) für Abgabestellen (Apotheken und Orthopädiefachgeschäfte) unterschiedlich regelt.

Leistungserbringer im Sinne des Krankenversicherungsgesetzes (KVG)

Grundvoraussetzung für die Tätigkeit zulasten der OKP ist, dass der jeweilige Leistungserbringer zugelassen ist.

Ärztinnen und Ärzte werden dann zugelassen, wenn sie das eidgenössische Diplom („Staatsexamen“) oder eine vom BAG anerkannte gleichwertige Ausbildung besitzen sowie über eine vom Bundesrat anerkannte Weiterbildung (Facharzttitel) verfügen (Artikel 36, Absatz 1 KVG).

Abgabestellen im Sinne von Artikel 55 Krankenversicherungsverordnung (KVV) sind insbesondere orthopädische Fachgeschäfte und Apotheken. Sie benötigen einen Vertrag mit den Leistungsträgern (Krankenkassen), damit verkaufte Produkte aus der MiGeL rückvergütet werden.

Artikel 20 der Krankenpflege-Leistungsverordnung (KLV) nennt als Leistungserbringer einzig Abgabestellen nach Artikel 55 KVV.

Als Grundsatzregelung schließt Artikel $20 \mathrm{KLV}$ jedoch nicht aus, dass ein ärztlich angeordnetes Mittel oder ein ärztlich angeordneter Gegenstand direkt vom zugelassenen, behandelnden Arzt abgegeben wird. Dieser Grundsatz wird durch ein Bundesgerichtsurteil (2010) gestützt.
Voraussetzungen für die Abgabe von Kompressionsstrümpfen in der gefäßmedizinischen Praxis einschließlich Rückvergütung durch die Krankenversicherung:

a) Fachwissen auf Seiten der Ärzteschaft und

b) Patient ist Endverbraucher des MiGeLProdukts.

Die Abgabe von Kompressionstrümpfen erfordert Fachwissen, über welches zugelassene Phlebolog*innen, Angiolog*innen und Gefäßchirurg*innen verfügen: fachgerechtes Maßnehmen, Anprobe und persönliche Beratung bezüglich Handhabung sowie regelmäßige Kontrollen. Endverbraucher und Anwender ist stets der Patient, allenfalls unter Hilfe seiner Angehörigen. Bei Bedarf kann eine mechanische Anziehhilfe abgegeben werden (MiGel Position 17.12.01).

Damit sind die Voraussetzung gegeben, dass gefäßmedizinisch spezialisierte Ärztinnen und Ärzte nach Artikel 20 Krankenleistungsverordnung (KLV) Kompressionsstrümpfe in der Praxis oder in der Institution, wo sie arbeiten, abgeben und nach MiGeL abrechnen können, sodass Patient* innen diese Auslagen von der Krankenversicherung zurückerstattet erhalten.

\section{Grundsätze der MiGeL und Kompres- sionsmittel innerhalb der MiGeL}

Die MiGeL ist eine abschließende PositivListe, d. h. nur auf der Liste aufgeführte Mittel und Gegenstände werden durch die OKP vergütet. Bei den in der MiGeL angegebenen Vergütungsbeträgen handelt es sich um Höchstpreise.

Die MiGeL enthält unter Position 17 eine Auflistung der zu vergütenden Kompressionstherapiemittel. Darunter fallen z. B. unter der Position 17.02 rund gestrickte 
medizinische Kompressionsstrümpfe und -strumpfhosen der Kompressionsklasse 2 (23-32 mmHg Andruck).

\section{Zusammenfassung}

Zum Schluss können wir noch einmal festhalten: Für die Abgabe respektive Verrechnung von Kompressionsstrümpfen zulasten der OKP durch die verschreibenden Ärzte ist aus juristischer Sicht kein Vertrag mit den Versicherern erforderlich.

\section{Remise de bas médicaux de compression en cabinet de médecine vasculaire Évaluation juridique par Me Patrizia Gratwohl, M.A. HSG in Law}

\section{Résumé (Jürg Hafner, Président SSP et Daniel Staub, Président USSMV)}

Les mensurations et la remise de bas médicaux de compression à charge de l'assurance obligatoire des soins (AOS) en cabinet de médecine vasculaire est une pratique incontestée en Suisse depuis des décennies.

Suite à l'entrée en vigueur de la révision de la liste des moyens et appareils (LiMA) le 1er avril 2019, le Comité de la SSP a été informé à plusieurs reprises que certaines caissesmaladie et autres assureurs-maladie refusaient de rembourser les bas médicaux de compression mesurés et remis en cabinet.

Afin d'examiner la situation, la SSP et I'USSVM ont chargé Me Patrizia Gratwohl de faire une évaluation juridique sous forme de mémorandum. Me Gratwohl, lic. en droit, est associée à l'étude d'avocats Steinbrüchel Hüssy Rechtsanwälte, 8001 Zurich.

Les lignes suivantes résument ce mémorandum qui nous a été remis le 1er mars 2021. II sera publié dans son intégralité sur nos sites Internet.

\section{MESSAGE PRINCIPAL}

Les médecins qui prescrivent des bas médicaux de compression ne sont pas tenus de conclure un contrat avec un assureur pour les remettre en cabinet et les facturer à l'AOS. Cela est directement lié à la loi qui réglemente de manière différenciée l'autorisation des produits de la LiMA pour (A) le corps médical et (B) les centres de remise (pharmacies et magasins orthopédiques).

Fournisseurs de prestations médicales au sens de la loi sur l'assurance-maladie (LAMal)

Le fournisseur de prestations médicales doit remplir les conditions édictées pour exercer une activité à charge de l'AOS.

Sont admis les médecins titulaires d'un diplôme fédéral (examen d'État) ou d'une formation équivalente reconnue par l'OFSP et d'une formation postgraduée reconnue par le Conseil fédéral (avec titre de spécialiste) selon l'art. 36, al. 1, LAMal.

Au sens de l'art. 55 de l'ordonnance sur l'assurance-maladie (OAMal), les centres de remise, principalement les magasins orthopédiques et les pharmacies, doivent avoir conclu un contrat avec les fournisseurs de prestations (caisses-maladie) pour que les produits vendus dans le cadre de la LiMA soient remboursés.

L'art. 20 de l'ordonnance sur les prestations de l'assurance des soins (OPAS) désigne uniquement les centres de remise comme fournisseurs de prestations au sens de l'art. 55, OAMal.

Cependant, selon cette règle de base de l'art. 20, l'OPAS n'exclut pas que le médecin traitant prescrive et remette directement un moyen ou un appareil en cabinet s'il remplit les conditions. Ce principe est confirmé dans un arrêté du Tribunal fédéral (2010).

Les conditions nécessaires à la remise de bas médicaux de compression en cabinet de médecine vasculaire, et à leur rembour- sement par l'assurance-maladie, sont les suivantes:

a) l'expertise reconnu du médecin fournisseur,

b) le patient est le destinataire final du produit selon la LiMA.

La remise de bas médicaux de compression nécessite une expertise que possèdent les phlébologues, les angiologues et les chirurgien.ne.s vasculaires agréé.e.s: mensurations correctes, essayage, conseils personnalisés en matière de manipulation, contrôles réguliers. Le destinataire et l'utilisateur final est toujours le patient, et ses proches s'il a besoin d'aide. Si nécessaire, il est possible de prescrire en sus une aide à l'enfilage (LiMA, pos. 17.12.01).

Ceci définit le cadre dans lequel les spécialistes en médecine vasculaire peuvent remettre des bas médicaux de compression dans leur cabinet ou dans l'établissement pour lequel ils travaillent conformément à l'art. 20 de l'ordonnance sur les prestations de l'assurance des soins (OPAS) et les facturer conformément à la LiMA, de sorte que les patientes et les patients puissent se faire rembourser cette dépense par leur assurance-maladie.

Principes de la LiMA et moyens de compression au sein de la LiMA

La LiMA est une liste positive exhaustive. Cela signifie que seuls les moyens et les appareils figurant sur cette liste sont remboursés par l'AOS. Les montants de remboursement spécifiés dans la LiMA sont des montants plafonds.

En position 17, la LiMA présente une liste des moyens de compression remboursables. En position 17.2, il s'agit par exemple des bas et collants médicaux de compression de la classe de compression 2 (23 à $32 \mathrm{mmHg}$ ) à maillage circulaire.

\section{Résumé}

En conclusion, nous pouvons une fois de plus l'affirmer: d'un point de vue juridique, les médecins qui prescrivent des bas médicaux de compression ne sont pas tenus de conclure un contrat avec un assureur pour les remettre en cabinet et les facturer à l'AOS. 